\title{
Investigation of Fully Three-Dimensional Helical RF Field Effects on TWT Beam/Circuit Interaction
}

\author{
Carol L. Kory, Senior Member \\ Analex Corporation \\ NASA Glenn Research Center \\ 21000 Brookpark Road, MS 54-5 \\ Cleveland, Ohio 44135 \\ 216-433-3512 Phone \\ 216-433-8705 Fax \\ ckory@grc.nasa.gov
}

\begin{abstract}
A fully three-dimensional (3D), time-dependent, helical traveling wave-tube (TWT) interaction model has been developed using the electromagnetic particle-in-cell (PIC) code MAFIA. The model includes a short section of helical slow-wave circuit with excitation fed by RF input/output couplers, and electron beam contained by periodic permanent magnet (PPM) focusing. All components of the model are simulated in three dimensions allowing the effects of the fully $3 \mathrm{D}$ helical fields on RF circuit/beam interaction to be investigated for the first time. The development of the interaction model is presented, and predicted TWT performance using 2.5D and 3D models is compared to investigate the effect of conventional approximations used in TWT analyses.
\end{abstract}

\section{INTRODUCTION}

The phenomenal growth of the satellite communications industry has created a large demand for TWTs operating with unprecedented specifications requiring design and production of many novel devices in record time. To achieve this, the TWT industry heavily relies upon computational modeling, which has advanced tremendously in the past few years. For example, Hughes Electron Dynamics recently implemented the helical modeling techniques developed in [i], resulting in strong advances toward first pass TWT design capabilities compared to typically three or four design-build iterations in the past [ii]. This has translated into major savings in development time and cost.

Still, there is a need for improvement in the TWT industry's computational modeling capabilities as there are often discrepancies between measured TWT data and that predicted by helical TWT interaction codes. To account for these discrepancies, 
"fudge factors" are incorporated where parameters such as dielectric constant, voltage, beam radius or attenuation are adjusted in the model until simulation matches experiment. The incorporation of these fudge factors for a class of TWTs provides reasonable results as long as the TWTs being modeled have similar geometries and operating characteristics. However, this severely limits the analysis and design of novel devices or TWTs with fairly different parameters. In addition, the inaccuracy of current computational tools, such as helical interaction models, limits achievable overall TWT efficiency because optimized designs require highly accurate models. In particular, collector efficiency, which significantly affects overall TWT efficiency, is difficult to optimize unless extremely accurate spent beam data is predicted by the interaction code.

Since the computational speeds of 2D/2.5D codes (minutes or even seconds per simulation) are invaluable for optimization where hundreds of runs must be made, it is necessary to remove the present limitations by developing more accurate $2 \mathrm{D} / 2.5 \mathrm{D}$ helical TWT interaction models. To do this, however, the sources of inaccuracy in existing models must clearly be defined requiring a fully $3 \mathrm{D}$ model without approximations.

The growth of the communications industry has also imposed a demand for increased data rates for transmission of large volumes of data. This requires minimum distortion of the modulated signal as it is passed through the TWT. To successfully minimize intersymbol interference, it is critical to correlate the quality of the transmitted data transmitted with TWT parameters. Unfortunately, limited experimental testing is available to perform this correlation, and until now a computational model did not exist. The time-domain helical TWT interaction model developed here, however, provides the capability to establish this computational test bench where signal integrity can be measured as a function of TWT operating parameters and component geometries, such as coupler configurations. Intermodulation products, harmonic generation and backward waves can also be monitored with the model for similar correlations. The advancements in computational capabilities and corresponding potential improvements in TWT linearity and efficiency may prove to be the enabling technologies for realizing unprecedented data rates for near real time transmission of increasingly larger volumes of data demanded by planned commercial and government satellite communications applications. 


\section{BACKGROUND}

It was demonstrated for the first time in [i] that using the eigenmode solver of the simulation code, MAFIA (Solution of MAxwell's equations by the Finite-IntegrationAlgorithm) [iii, iv], 3D cold-test (no electron beam present) helical structures can be accurately modeled with actual tape width and thickness, dielectric support rod geometry and materials. This accurate helical cold-test model was used to investigate standard approximations currently used in helical TWT interaction codes [v]. In particular, these codes typically assume the nonfundamental longitudinal and azimuthal space harmonics, $\mathrm{n}$ and $\mathrm{v}$, respectively, of the longitudinal component of the $\mathrm{RF}$ electric field, $\mathrm{E}_{\mathrm{z}}$, are zero. They also assume that the azimuthal component of the RF electric field, $\mathrm{E}_{\theta}$, is zero. From here forth, these will be referred to as the approximations in question. Using the MAFIA eigenmode solver helical cold-test model, these conventionally used approximations were found to be in significant error. $E_{\theta}$ and the $n=v=-1$ longitudinal and azimuthal space harmonics of $\mathrm{E}_{\mathrm{z}}$ were found to have significant amplitudes (compared to the fundamental). This is especially a concern near the output of the TWT where RF fields have grown substantially due to the transfer of energy from the electron beam. To estimate the significance these RF fields have at the TWT output, the electric fields obtained using the eigenmode solver model were scaled to be commensurate with those at the output of the Hughes $8916 \mathrm{H}, 18-40 \mathrm{GHz}$ helical TWT, which was also used as a model for this study. The forces associated with these scaled RF fields were compared to the electron beam radial space charge forces since electron beam focusing structures are typically designed using beam optics codes based predominantly on balancing these forces. The forces at the beam radius due to radial, azimuthal and longitudinal RF electric fields at the TWT output were found to reach $61 \%, 26 \%$ and $132 \%$ of radial space charge forces, respectively, at $26 \mathrm{GHz}$ for the device studied [vi].

These results imply that implementing the approximations in question will significantly affect TWT performance, and contribute to discrepancies between experimental TWT data and that predicted by conventional TWT helical interaction codes. These RF field characteristics also imply azimuthally dispersive spent beam data, where conventional codes assume an azimuthally symmetric distribution. 
Discussing qualitatively an estimate of the effects these approximations may have on TWT operation, we would expect the presence of $E_{\theta}$ to have an influence on beam rotation. As a preliminary investigation, the rotational forces on the beam due to both the peak radial component of the magnetic focusing field and the RF $E_{\theta}$ at the $8916 \mathrm{H}$ TWT output were calculated. Results showed that the azimuthal forces from the magnet stack for Brillouin focusing are about 60 times larger than the forces from the RF $E_{\theta}$. This indicates that the effect of $\mathrm{E}_{\theta}$ may be negligible; however, when immersed in periodic focusing fields, the beam will alternately rotate in the clockwise and counterclockwise directions, ceasing rotation in between. If the beam is exposed to the maximum value of $E_{\theta}$ at the point when the beam stops rotation, the azimuthal force from $E_{\theta}$ could have a significant effect. Additionally, there is the possibility that the rotating beam will excite a mode associated with $\mathrm{E}_{\theta}$.

The presence of the $n=-1$ space harmonic of $E_{z}$ implies the existence of waves propagating in the backward direction. This space harmonic of $\mathrm{E}_{\mathrm{z}}$ is infamous for its interaction with the electron beam resulting in backward wave oscillations (BWOs). The length of the interaction structure of this study, however, is most likely not long enough for these phenomena to appear. Incorporation of the $n=-1$ harmonic is extremely valuable in BWO analysis, however, and this model will be used extensively for this purpose when the interaction length is increased.

The presence of the $\mathrm{v}=-1$ space harmonic of $\mathrm{E}_{\mathrm{z}}$ indicates that $\mathrm{E}_{\mathrm{z}}$ varies with the azimuthal coordinate. This also implies that bunching will not occur uniformly across the beam cross section, but it is unclear whether this effect will be large enough to disrupt the exchange of energy between the beam and the RF wave. Again, there is a possibility that the rotating beam will excite this mode.

Quantitatively determining the effects of the approximations in question on predicted TWT performance is a highly complex and nonlinear process, requiring a fully $3 \mathrm{D}$ interaction model to accurately represent the RF fields and the corresponding beam behavior. Therefore, to determine their significance on predicted TWT performance, this work describes the development of a fully 3D helical TWT interaction model and a comparison of its results to the conventional 2.5D helical interaction code TWA3, which incorporates the approximations in question. The 3D model includes the helical slow- 
wave circuit, input/output couplers, and electron beam contained by PPM focusing.

MAFIA is a modular, time-dependent, particle-in-cell (PIC) code. The modules used in this study include M (mesh generator), S (static solver), E (eigenmode solver), T3 (3D time domain solver), TS3 (3D PIC solver) and P (postprocessor). The Finite Integration Technique (FIT) algorithm produces a matrix of finite-difference equations for electric and magnetic field vectors in the structure under study. The solution of these equations yields static, frequency-domain or time-domain solutions of Maxwell's equations. Thus, the full $3 \mathrm{D}$ representation of fields and corresponding spatial harmonics are present. The PIC solver computes the time-integration of electromagnetic fields simultaneously with the time integration of the equations of motion of charged particles that move under the influence of those fields. Fields caused by those moving charges are also taken into account; thus, effects like space charge and magnetic forces between particles are fully simulated [vii]. Particle motion is unrestricted, so particle trajectories can cross paths and move in three dimensions under the influence of $3 \mathrm{D}$ electric and magnetic fields. Correspondingly, there is no limit on the current density distribution of the electron beam.

TWA3 is a $2.5 \mathrm{D}$ (radial (r) and longitudinal (z) RF field components and $\mathrm{r}$ and $\mathrm{z}$ beam motion, with the addition of azimuthally symmetric angular velocity), nonlinear, multisignal, helical TWT, interaction code [viii]. A Lagrangian formulation is used to represent the electron beam with a deformable ring model, allowing the rings to move radially and overlap if the beam becomes non-uniform in cross-section. The axial velocity can vary between rings as well. When TWA3 is run, the time and radius are calculated for each disk as it passes a longitudinal position along the TWT. Data is obtained over one or more RF periods as a function of longitudinal position and not as a function of time; hence, the code is referred to as steady-state. All spatial harmonics of $\mathrm{E}_{\mathrm{z}}$ except the fundamental $(\mathrm{n}=\mathrm{v}=0)$ are neglected.

The TWT used as a model is a 40 Watt, $18-40 \mathrm{GHz}$ TWT for the millimeter-wave power module (MMPM) designated the Hughes $8916 \mathrm{H}$. The TWT includes a rectangular tape, helical, slow-wave circuit and periodic permanent magnet (PPM) focusing. Coaxial and waveguide couplers are used to couple the RF signal in and out of the TWT, respectively. The operating parameters for the TWT at mid-band are shown in Table I. 
Table I Operating parameters for Hughes $8916 \mathrm{H}$ helical TWT at mid-band

\begin{tabular}{|l|l|}
\hline Beam voltage $\mathbf{V}_{\mathbf{o}}(\mathbf{k V})$ & 7.6 \\
\hline Beam current $\mathbf{I}_{\mathbf{0}}(\mathbf{m A})$ & 81.0 \\
\hline Input power $\mathbf{P}_{\text {in }}(\mathbf{m W})$ & 25.0 \\
\hline Output power $\mathbf{P}_{\text {out }}(\mathbf{W})$ & 46.0 \\
\hline Initial beam radius/average helix radius $\mathbf{b} / \mathbf{a}$ & 0.5 \\
\hline
\end{tabular}

\section{MAFIA 3D INTERACTION MODEL}

There are four major obstacles to overcome in the development of the fully $3 \mathrm{D}$, PPM focused, helical TWT, interaction model using MAFIA. These are the development and validation of the: 1) PPM focusing model, 2) Electron optics model (including electron beam focused by the static fields from the PPM stack), 3) Helical slow-wave circuit model and input/output coupler model to couple the RF signal in and out of the helical circuit and 4) Helical TWT interaction model. Typically, 2D or 2.5D TWT interaction codes represent the entire slow-wave structure (over 100 helical turns or cavities) with severs (included to prevent backward wave oscillations), and velocity taper (geometric tapering of the slow-wave circuit to prolong synchronism between the beam and the RF wave typically implemented at the end of the TWT). Because of the computational intensity added by modeling in $3 \mathrm{D}$, however, the interaction model of this study is limited to a short section of a TWT consisting of up to about 40 helical turns. This length is sufficient for this study where the effects of the 3D RF fields on TWT characteristics are investigated.

\section{III.1 PPM focusing model}

Actual dimensions and material properties of the periodic permanent magnet focusing stack for the Hughes $8916 \mathrm{H}$ TWT [ix] were modeled using MAFIA. The focusing stack is a sequence of iron pole pieces and opposite polarity samarium cobalt $\mathrm{Sm}_{2} \mathrm{Co}_{17}$ magnets. The provided $\mathrm{B}-\mathrm{H}$ curve $[\mathrm{x}]$ was used in the MAFIA static solver for the iron pole pieces and a fixed magnetization and permeability were used for the magnets. The magnetic flux density was calculated and compared with experimental data 
with excellent agreement [xi].

\section{III.2 Electron optics model}

The 3D PIC solver of MAFIA was used to simulate an electron beam immersed in the previously calculated static magnetic fields from the PPM stack. 20000 particles per $\mathrm{RF}$ period were used for all simulations, unless otherwise indicated. The MAFIA 3D electron optics model was compared to the classic analysis by Mendel et al. [xii] and reported on in [xi]. It was also used to investigate the effect of azimuthally asymmetric PPM stack characteristics on electron beam behavior in [xiii].

\section{III.3 Helical slow-wave circuit and input/output coupler model}

Using the time-domain solver of MAFIA, several turns of the helical slow-wave circuit were modeled with couplers to feed the excitation signal in and out of the structure. The $8916 \mathrm{H}$ helix TWT slow-wave circuit used as a model for this study includes rectangular, tungsten, helical tape supported by $\mathrm{T}$-shaped $\mathrm{BeO}$ rods inside a conducting barrel (See Figure 1). The model excludes the electron beam; thus, it is a cold-test model similar to that using the eigenmode solver of MAFIA described in [i]. The coupler design and time-domain simulation procedure is described followed by a comparison between the time-domain and already validated eigenmode helical cold-test models.

\section{III.3.1 Coupler design}

Actual coupling elements for the $8916 \mathrm{H}$ TWT are highly complex because they must achieve a match over a large bandwidth, and their geometries are quite large relative to the helical circuit. To reduce computational complexity, the coupler configurations of this study are based on a simple coaxial cable with center conductor equivalent to the helical tape of the $8916 \mathrm{H}$ TWT to minimize mismatch. The structure is modeled as a symmetric network with input and output couplers having equivalent dimensions as shown in Figure 1 where a cutaway 3D view of several helical turns of the structure is shown.

To calculate the S-parameters with MAFIA, 2D mesh planes are created at the cross-sections of the input and output waveguides and the eigenmode solver is used to 
compute the input/output waveguide modes at a particular frequency. These modes are loaded into the 3D time-domain solver at the waveguide boundaries to simulate infinitely long waveguides beyond the computational space. The input waveguide is excited and the signals at the input and output ports are monitored during the time-integration. When the simulation has reached steady-state, the amplitudes and phases of the signals are used to calculate the S-parameters.

It is necessary to use a large number of mesh cells when modeling the helical circuit because of its complexity; correspondingly, the computational time and memory increase significantly with number of helical turns. Thus, the number of turns modeled was limited in this study to about 20, and in some cases about 40. (Figure 1 shows only about 10 turns to provide a clear illustration of the modeled structure).

The voltage standing wave ratio (VSWR), calculated as

$$
V S W R=\frac{1+\left|S_{11}\right|}{1-\left|S_{11}\right|},
$$

is plotted versus several frequency points for the final coupler design in Figure 2. $\left(\mathrm{S}_{11}\right.$ is the ratio of the amplitudes of the reflected and input signals at the input port). The match is excellent at mid-band with a VSWR of 1.04, but worsens as the frequency is varied with a VSWR of almost 1.5 at $40 \mathrm{GHz}$.

\section{III.3.2 Comparison of time-domain and eigenmode cold-test helical models}

To validate the time-domain helical slow-wave circuit and RF coupler model, the dispersion and interaction impedance were calculated and compared to the already validated $8916 \mathrm{H}$ helical eigenmode cold-test model [v]. Using the time-domain model, the phase shift per turn $\beta_{0} \mathrm{p}$ (where $\beta_{0}$ is the fundamental space harmonic of the axial propagation constant and $\mathrm{p}$ is the helical pitch) is determined from the phase shift per turn of the longitudinal electric field on the helical axis averaged over one RF period. The phase velocity is calculated as

$$
v_{p}=\frac{\omega}{\beta_{0}}=\frac{2 \pi f p}{\beta_{0} p} .
$$

where $\mathrm{f}$ is the excitation frequency. The cold-test dispersion obtained using the eigenmode and time-domain solvers of MAFIA is compared to measured results in 
Figure 3 showing very good agreement. Compared to experimental data, the absolute average differences across the bandwidth are 0.8 and 0.13 for the time-domain and eigenmode solver models, respectively. The difference between the time-domain and eigenmode models is most likely due to the difference in mesh resolution. The resolution of the time-domain model is necessarily more course to conserve computational time and memory. Including the couplers and about 20 helical turns, a resolution in the Cartesian coordinate system of $49 \times 40 \times 399$ in the $\mathrm{x}, \mathrm{y}$ and $\mathrm{z}$ directions, respectively, was used for the time-domain simulations. The eigenmode model resolution for one helical turn was $67 \times 67 \times 19$.

The on-axis interaction impedance of the fundamental space harmonic $\mathrm{K}_{0}$ is defined as

$$
\mathrm{K}_{0}=\frac{\left|E_{z 0}(0)\right|^{2}}{2 \beta_{0}^{2} P}
$$

where $\left|E_{z 0}(0)\right|$ is the magnitude of the fundamental space harmonic of the on-axis RF longitudinal electric field and $\mathrm{P}$ is the total RF power flow. Using the time-domain model, $\left|E_{z 0}(0)\right|$ is determined by averaging this value over one RF period, and the power is determined by the input excitation power of each simulation. The on-axis interaction impedance using the time-domain model is compared to the data obtained using the eigenmode model in Figure 4. The absolute average difference across the bandwidth between the time-domain and eigenmode models is 0.6 percent. It was shown in [v] that a more accurate interaction impedance is achievable using the 3D MAFIA eigenmode cold-test model compared to experimental perturbation methods; thus, experimental data are not shown.

\section{III.4 Helical TWT interaction model}

To complete the helical interaction model, the separate elements discussed thus far are joined using the MAFIA 3D PIC solver. About 20 helical turns were modeled with input/output coupling as described in Section III.3. Both the PPM stack magnetic flux density from the static solver and the waveguide boundary modes from the eigenmode solver were loaded at the onset of the simulation. Then, the beam and RF drive were simultaneously "turned on". Each was linearly ramped in excitation over five 
RF periods to reduce turn-on transients and run for several additional RF periods at peak values. Figure 5 shows the time-dependent signals representing the input, $V_{\text {in }}$, output, $\mathrm{V}_{\text {out }}$, and reflected signal at the input port, $\mathrm{V}_{\text {inref, }}$, for a simulation with $\mathrm{P}_{\text {in }}=25 \mathrm{~mW}, \mathrm{f}=$ $29 \mathrm{GHz}$, and beam radius to average helix radius ratio, b/a, of 0.5 . Strictly, these timedependent signals represent the square root of the peak power, or $\sqrt{2 P_{i n}}$. A turn-on transient exists, but at $t_{\text {gain }}$ (see Figure 5) where the TWT output parameters are calculated, only the reflected portion of this transient will be present in the system with oscillation commensurate with the ramped portion of the input signal. Therefore, the effect of the transient at this point in time will manifest itself predominantly as a DC bias in $\mathrm{V}_{\text {out }}$. To account for this bias, the average peak value of $\mathrm{V}_{\text {out }}$ is taken over one $\mathrm{RF}$ period starting at $t_{\text {gain }}$.

The CPU time for the 20 turn model is about 22 hours on a Sun Ultra SPARC 60 workstation for a single frequency case, run for $15 \mathrm{RF}$ periods.

\section{COMPARISON OF 2.5D AND 3D INTERACTION MODELS}

The 3D MAFIA helical interaction model is compared to the 2.5D model TWA3 [viii] to demonstrate the differences in predicted TWT operation between a $2.5 \mathrm{D}$ code incorporating the approximations in question and a $3 \mathrm{D}$ model that fully represents the helical fields. It was shown in $[\mathrm{v}]$ that the accuracy of the approximations in question worsens with increasing beam diameter and increasing $\beta_{0}$ p. For example, interpolating data from Figure 7 and Figure 9 of [v], where the relative strengths of the $n=-1$ and $v=-$ 1 space harmonics are plotted, respectively, the $n=v=-1$ space harmonics of $E_{z}$ at 29 $\mathrm{GHz}$ are about 14 percent of the fundamental for a beam radius to average helix radius ratio (b/a) of 0.25 increasing to about 34 percent for $b / a=0.5$. Accordingly, if the approximations in question have a significant effect on TWT operation, we would expect them to appear as a discrepancy between $2.5 \mathrm{D}$ and $3 \mathrm{D}$ codes, increasing with increasing beam diameter and increasing RF input drive power (where the relative RF field strength is also increasing).

Equivalent helical length, phase velocity, interaction impedance, attenuation (assumed to be zero), focusing conditions and RF drive were used in the 2.5D TWA3 and 
3D MAFIA models. An $81 \mathrm{~mA}, 7600 \mathrm{~V}$ beam and a $29 \mathrm{GHz}$ excitation signal were used for all simulations. To clearly illustrate RF field effects, the simulations utilize an electron beam without initial transverse velocities and magnetic focusing strength approximately equal to the Brillouin field so that beam perturbations will be predominantly due to $R F$ field forces. Simulations were completed for $b / a=0.1,0.25$, 0.5 and 0.7 ; thus, it was necessary to recalculate the magnetic flux density for each beam diameter to maintain a focusing strength proportional to the Brillouin field.

\section{IV.1 20 turn model $\left(P_{i n}=0 \mathrm{~W}\right)$}

First, the MAFIA and TWA3 models are compared without RF drive to validate the equivalence of the electron optics portions of the codes. Simulations were completed for a beam having $\mathrm{b} / \mathrm{a}=0.5$ with various focusing field strengths. The percent ripple and percent transmission are compared for MAFIA and TWA3 in Figure 6 and Figure 7, respectively, versus field strength normalized to the Brillouin field. The agreement is excellent confirming the agreement between the electron optics portions of the codes.

\section{IV.2 20 turn model $\left(P_{\text {in }}=25 \mathrm{~mW}\right)$}

Next, an input power equivalent to the input power of the experimental $8916 \mathrm{H}$ TWT ( $25 \mathrm{~mW}$ ) was used to drive both the MAFIA and TWA3 models using beam radii of $0.1,0.25,0.5$ and 0.7 of the average helix radius. When $P_{\text {in }}=25 \mathrm{~mW}$ the RF field forces are small relative to space charge and magnetic focusing forces; however, the amplitudes of the non-fundamental RF space harmonics, particularly the $\mathrm{n}=\mathrm{v}=-1$ harmonics, will have the same relative strength compared to the fundamental harmonic regardless of the excitation power. Accordingly, one would still expect discrepancies between the results predicted by the $2.5 \mathrm{D}$ and $3 \mathrm{D}$ codes.

The gain and efficiency obtained from TWA 3 and MAFIA are compared in Figure 8 and Figure 9, respectively, showing an absolute average gain difference of 0.44 $\mathrm{dB}$. The differences increase slightly with beam radius. The gain is very sensitive to the synchronism between the RF circuit and the electron beam; thus, the discrepancy in gain may be caused by the difference in how the beam velocities are modeled between codes and the corresponding hot phase velocity of the RF circuit. Increasing the phase velocity by only 0.78 percent in the TWA 3 simulations results in a comparable gain to the 
MAFIA predicted value at $\mathrm{b} / \mathrm{a}=0.1$. We make the phase velocity adjustment to match the gain at this beam radius since the approximations in question have a small effect at very small beam radii. The TWA3 gain and efficiency values with adjusted phase velocities are also shown in Figure 8 and Figure 9, respectively. Again, there is a slight but steadily increasing difference between codes with increasing beam diameter with an absolute average gain difference of $0.04 \mathrm{~dB}$. The percent transmission is 100 percent for all simulations for both models. The adjusted phase velocity was used in the TWA3 simulations for all subsequent simulations.

\section{IV.3 20 turn model $\left(\mathrm{P}_{\text {in }}=46 \mathrm{~W}\right)$}

Next, an input power equivalent to the output power of the experimental $8916 \mathrm{H}$ TWT (46 W) was used to drive both the TWA3 and MAFIA models to compare the performance at high power levels (as would occur at the output of the TWT) where RF fields are relatively large. Unlike the actual TWT output, however, the beam is unmodulated at the simulation input because of computational limitations. Still, the simulations demonstrate the effect of the RF fields commensurate with the TWT output compared with those at the input.

When $\mathrm{P}_{\text {in }}=46 \mathrm{~W}$ the RF field forces are significant compared to magnetic focusing and space charge forces. Thus, one would expect the effects of the nonfundamental space harmonics and $E_{\theta}$ to increase in significance compared to the case where a low RF drive is used, and as a result the discrepancies between $3 \mathrm{D}$ and $2.5 \mathrm{D}$ codes to increase as well. The MAFIA 2D beam profiles immersed in the dynamic electric fields at several time steps over one RF period are shown for the case where b/a = 0.5 in Figure 10, illustrating the electron beam traveling in synchronism with the RF wave. Note, interception between the beam and the helix occurs toward the last few helical turns due to the strong defocusing of the RF radial fields.

The gain is compared for each code in Figure 11. Good agreement is shown at $\mathrm{b} / \mathrm{a}=0.1$ where the approximations in question are least significant, and the differences in gain between the codes increase with beam diameter until $b / a=0.7$. At this diameter, significant interception of the beam is occurring, and consequently, less beam current interacts with the RF fields accounting for the deviation in gain differences between 
codes. The absolute average difference in gain is $0.15 \mathrm{~dB}$ compared to $0.04 \mathrm{~dB}$ when $\mathrm{P}_{\text {in }}$ $=25 \mathrm{~mW}$. The increasing differences between codes with increasing beam diameter and increasing RF power imply that the approximations in question are responsible for the discrepancies.

The percent transmission is compared for each code in Figure 12. The values from MAFIA and TWA3 are almost identical, implying that the approximations in question have little effect on the TWT percent beam transmission using the current model.

\section{IV.4 40 turn model $\left(\mathrm{P}_{\text {in }}=25 \mathrm{~mW}\right)$}

Although the average absolute differences in gain between MAFIA and TWA3 for the simulations where $\mathrm{P}_{\text {in }}=25 \mathrm{~mW}$ (without adjusting the TWA3 phase velocity) was only approximately $0.4 \mathrm{~dB}$, this corresponds to an average percent difference of about 45 percent. To investigate how the codes compare with a longer helical section, the MAFIA model was increased from 20 to 40 helical turns. The mesh was necessarily reduced for computational reasons from $53 \times 43 \times 399$ to $49 \times 40 \times 759$, in the $\mathrm{x}, \mathrm{y}$ and $\mathrm{z}$ directions, respectively. Initially, the 20 turn case was run with a reduced number of particles from $20,000 /$ RF period to $10,000 / R F$ period and the gain and efficiency calculated for $P_{\text {in }}=25$ $\mathrm{mW}$ and $\mathrm{b} / \mathrm{a}=0.5$. The results for each case were very similar; therefore, 10,000 particles per RF period were used in the 40 turn simulations.

With the above simplifications, the MAFIA 40 helical turn model was initially run without the electron beam and the VSWR, phase velocity and impedance were calculated. The cold-test data were identical to that calculated using the 20 turn model. Next, the model was run with $\mathrm{P}_{\text {in }}=25 \mathrm{~mW}$ and $\mathrm{b} / \mathrm{a}=0.5$. The difference in gain between MAFIA and TWA3 was $0.38 \mathrm{~dB}$, or 4.1 percent. Thus, we can conclude that the interaction model using MAFIA provides similar results to TWA3 even with a longer interaction length.

Keeping $\mathrm{b} / \mathrm{a}=0.5$, the 40 turn model was run with varying input drive power to investigate how the $2.5 \mathrm{D}$ and $3 \mathrm{D}$ codes compare with increasing $\mathrm{RF}$ field strength. The calculated gain and output power are plotted versus input power in Figure 13 and Figure 14 , respectively, showing the 40 turn interaction section has been driven close to saturation. There is an increasing difference between codes with increasing input drive 
power (and increasing RF field strength), again implying the approximations in question cause the discrepancies. Interception of the beam with the helix occurred for simulated input powers of $10 \mathrm{~W}$ and greater, and since more of the beam is intercepted at $\mathrm{P}_{\text {in }}=20$ $\mathrm{W}$, there is less beam current interacting with the RF fields and a corresponding decrease in the discrepancy between codes. The average absolute difference in gain between the $3 \mathrm{D}$ and $2.5 \mathrm{D}$ models is $0.87 \mathrm{~dB}$. The CPU time for each simulation was about 36 hours.

For all simulations, the gain data using the $2.5 \mathrm{D}$ helical interaction model was consistently lower than that predicted by the $3 \mathrm{D}$ model. Wilson has found that there is a general tendency for $2.5 \mathrm{D}$ codes to underestimate saturated gain compared to experiment [xiv]. These results further support the presumption that the approximations in question contribute to the discrepancies between measured and simulated data from 2.5D helical interaction codes.

\section{CONCLUSIONS AND FUTURE WORK}

A fully $3 \mathrm{D}$ helical traveling wave tube interaction model was developed including a short section of helical slow-wave circuit, time-dependent excitation introduced and removed by RF input/output couplers, respectively, and electron beam contained by PPM focusing. The effects of approximations made in conventional helical interaction codes on RF circuit/beam interactions were investigated by comparing $3 \mathrm{D}$ simulations to a conventional 2.5D interaction code TWA3. The results from these simulations imply there is a definite effect that manifests itself as an increasing difference between $2.5 \mathrm{D}$ and $3 \mathrm{D}$ codes with increasing beam diameter (where the approximations in question grow less accurate) and with increasing RF input power (where the effect of the approximations will be more significant). Results showed $2.5 \mathrm{D}$ simulations consistently predicted gains lower than that predicted by the 3D model. Since there is also a general tendency for 2.5D codes to underestimate saturated gain compared to experiment [xiv], this further supports the presumption that the approximations in question contribute to the discrepancies between measured and simulated data from 2.5D helical interaction codes.

For the purpose of this work, a model without any simplifications was necessary; however, the computational intensity limited the modeled length of the slow-wave 
circuit. In the future, the model will be simplified allowing the length of the structure to be increased so that simulations can be compared to measured data. This will also allow interaction of the electron beam with backward waves to be monitored, permitting the computational capability to characterize the dependence of backward waves on 3D TWT geometries.

This time-dependent 3D helical interaction model is also the foundation for other areas of research. The TWT is commonly used as a power amplifier for satellite communications, which demand high data rates for near real time transmission of large volumes of data. The requirements placed on the TWT to obtain these data rates highly depend on the number of carriers and modulation scheme used in the input signal. For example, when multiple carrier signals are to be amplified, operating in the nonlinear region of the TWT causes carrier distortion; therefore, it is desired to back off the input power so the TWT operates in the linear region. Typically, as the input drive is backed off from saturation, the variation in gain with frequency, or gain ripple, also increases. This was found to be particularly disruptive to an amplified serial minimum shift keying (SMSK) signal when bit-error-rate (BER) measurements were conducted at NASA Glenn Research Center $[\mathrm{xv}]$. For this study, a simple sinusoidal RF excitation signal was used in the MAFIA model. A user defined signal can be supplied, however, so simulated investigations of digital data transmission capabilities of the TWT (BER testing) can be computationally conducted allowing one to correlate signal degradation imposed by the TWT with variations in TWT geometry and operating parameters. These modeling capabilities may prove to be the enabling technology in realizing unprecedented data rates.

Three-dimensional modeling of electron guns and collectors has been accomplished in the past. The development of an accurate 3D interaction model completes the validation of the major components of the TWT setting the stage for $3 \mathrm{D}$ simulations of the entire TWT. Although computational capabilities limit the realization of this goal at present, ever-improving computer resources promise realization in the near future. 


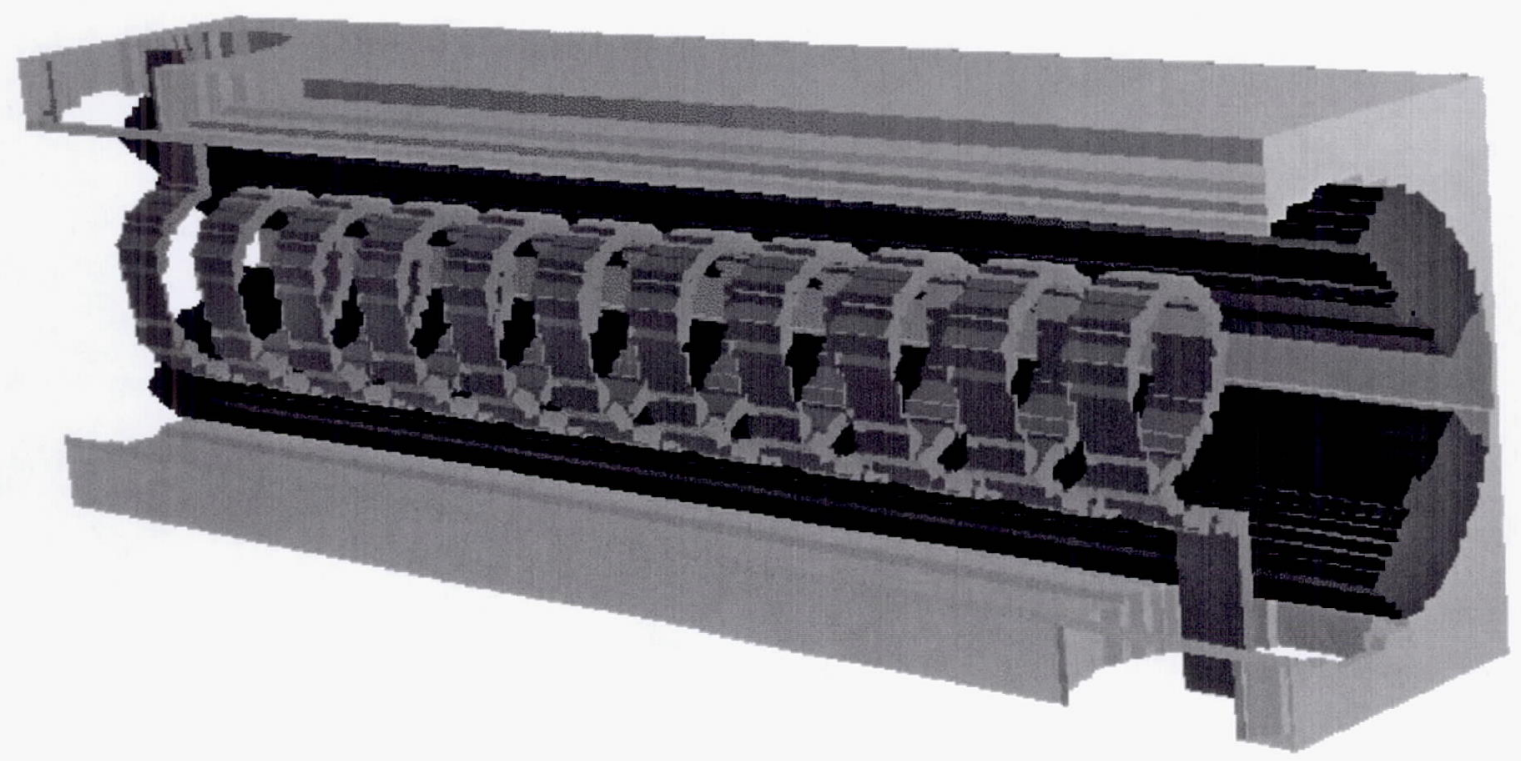

Figure 1 Cutaway of several turns of the helical slow-wave circuit with input/output couplers

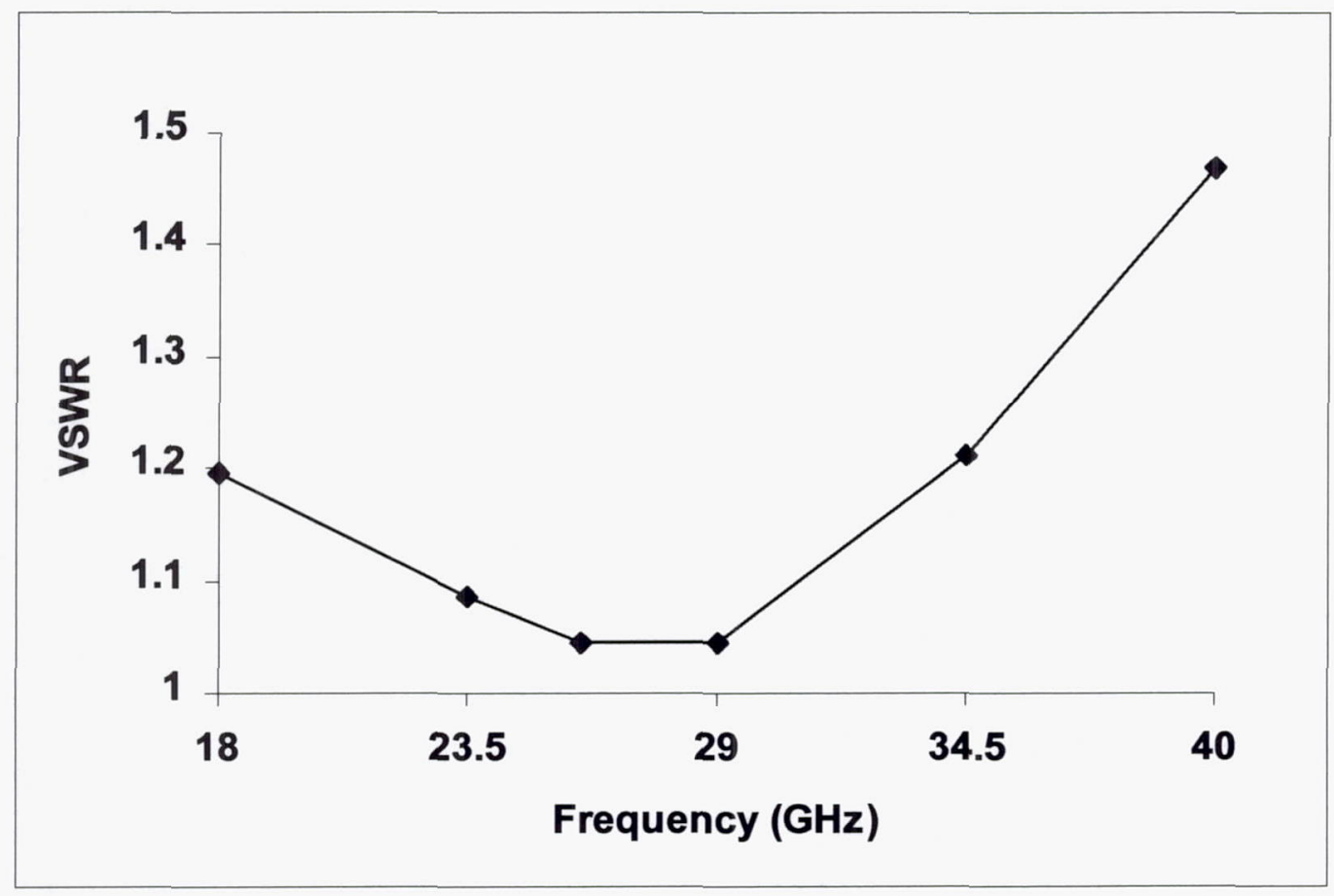

Figure 2 VSWR as a function of frequency for coupler design shown in Figure 1 


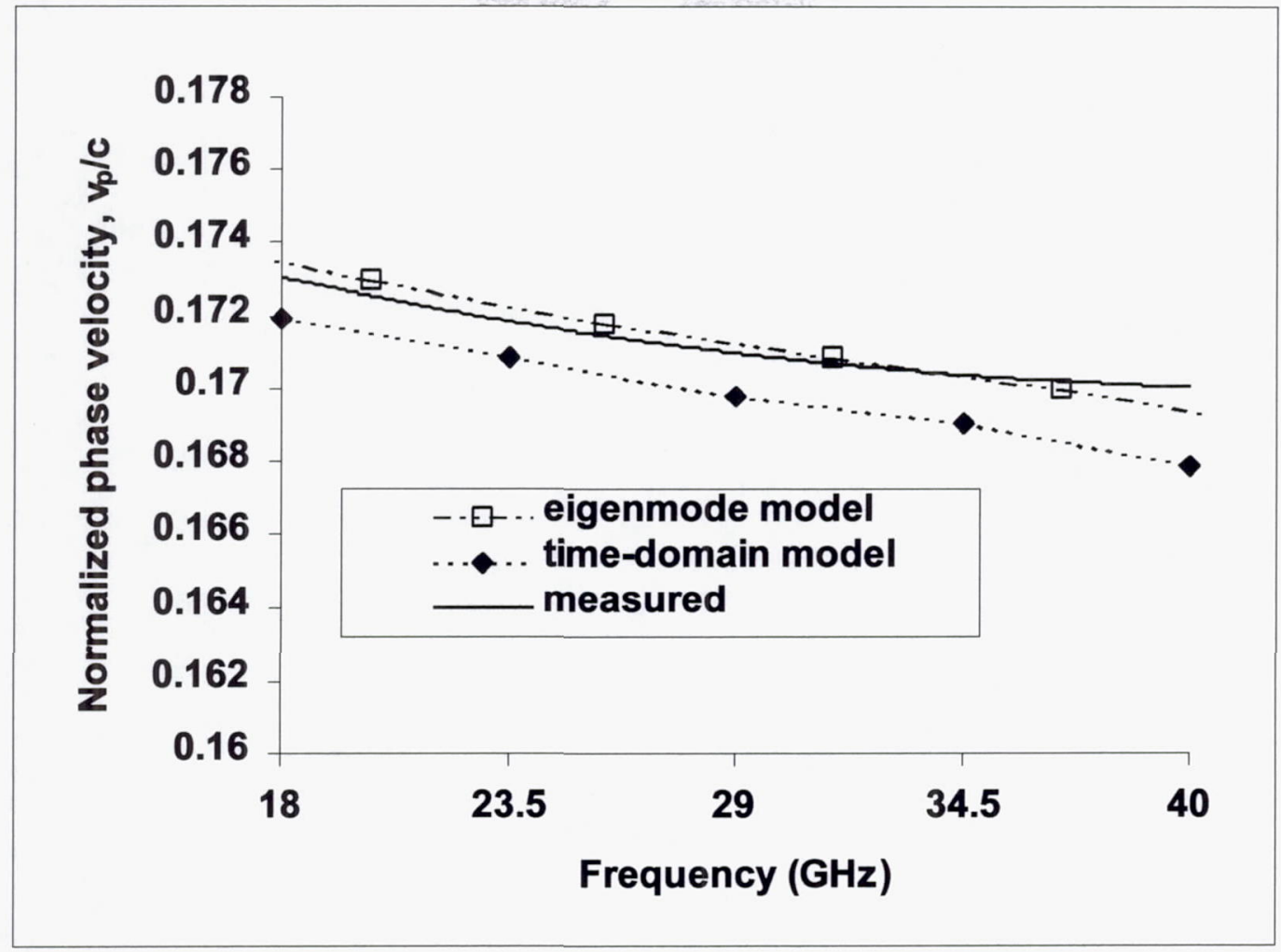

Figure 3 Measured and simulated cold-test dispersion for $8916 \mathrm{H}$ helical TWT slowwave circuit using eigenmode and time-domain models 


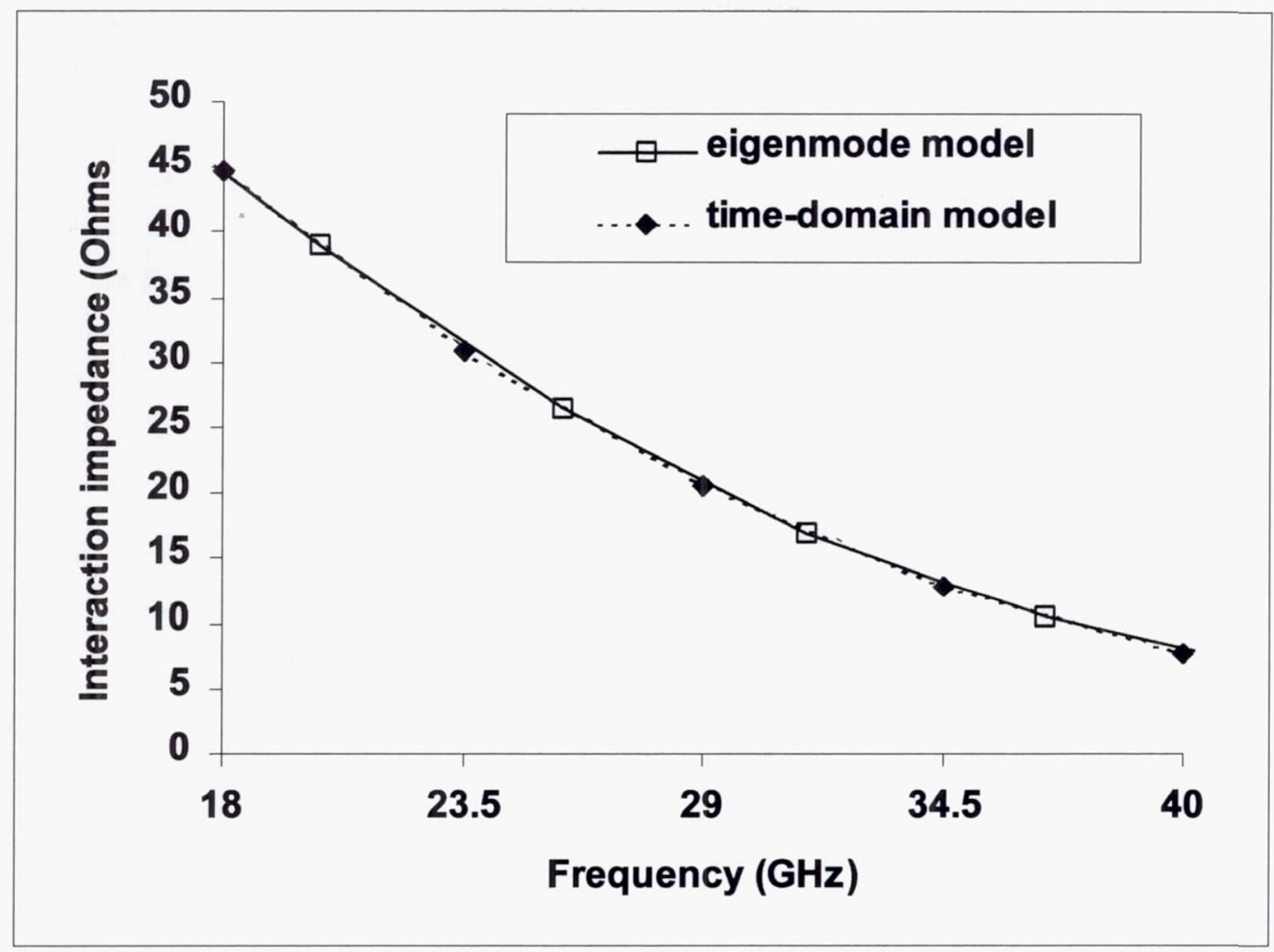

Figure 4 Simulated cold-test on-axis interaction impedance for $8916 \mathrm{H}$ helical TWT slow-wave circuit using eigenmode and time-domain models 


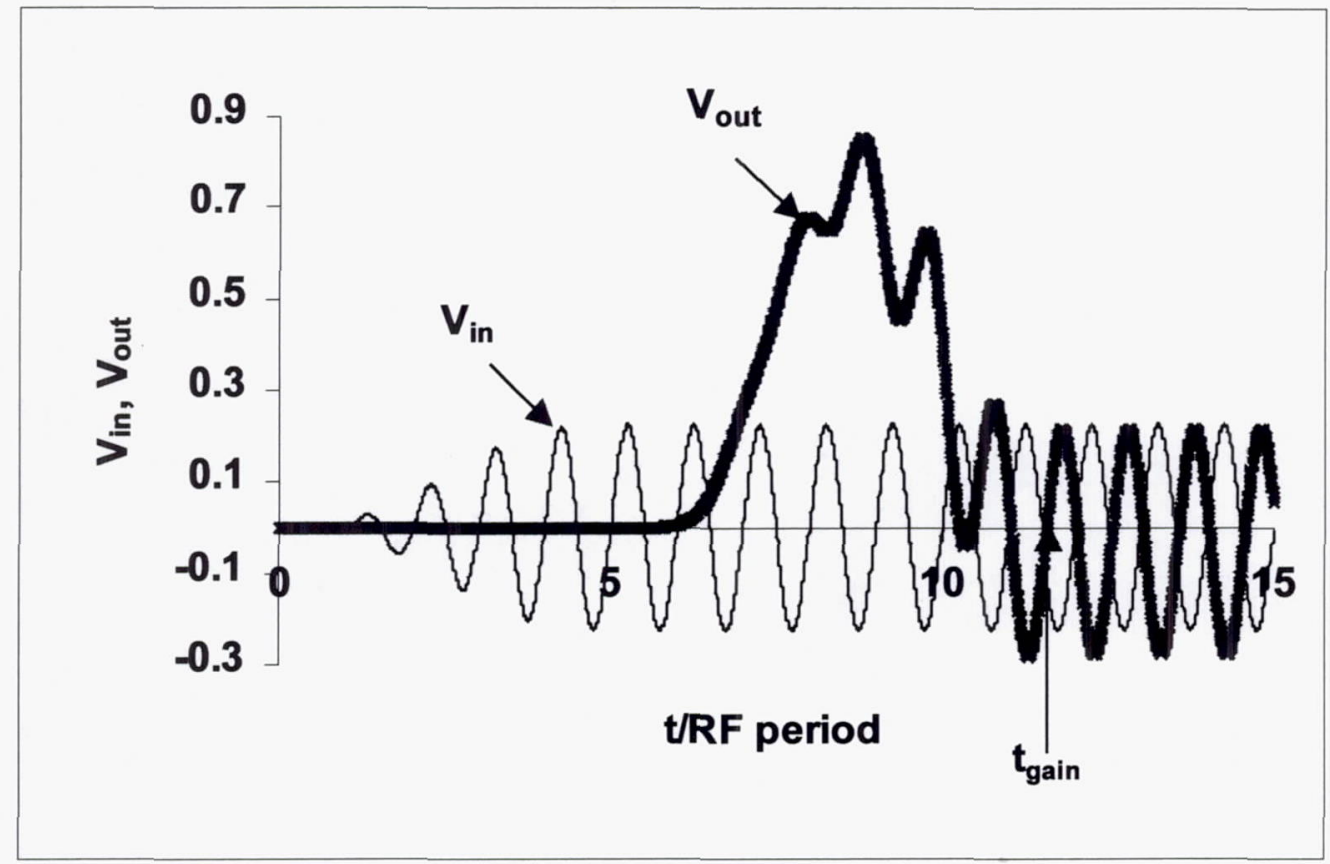

(a)

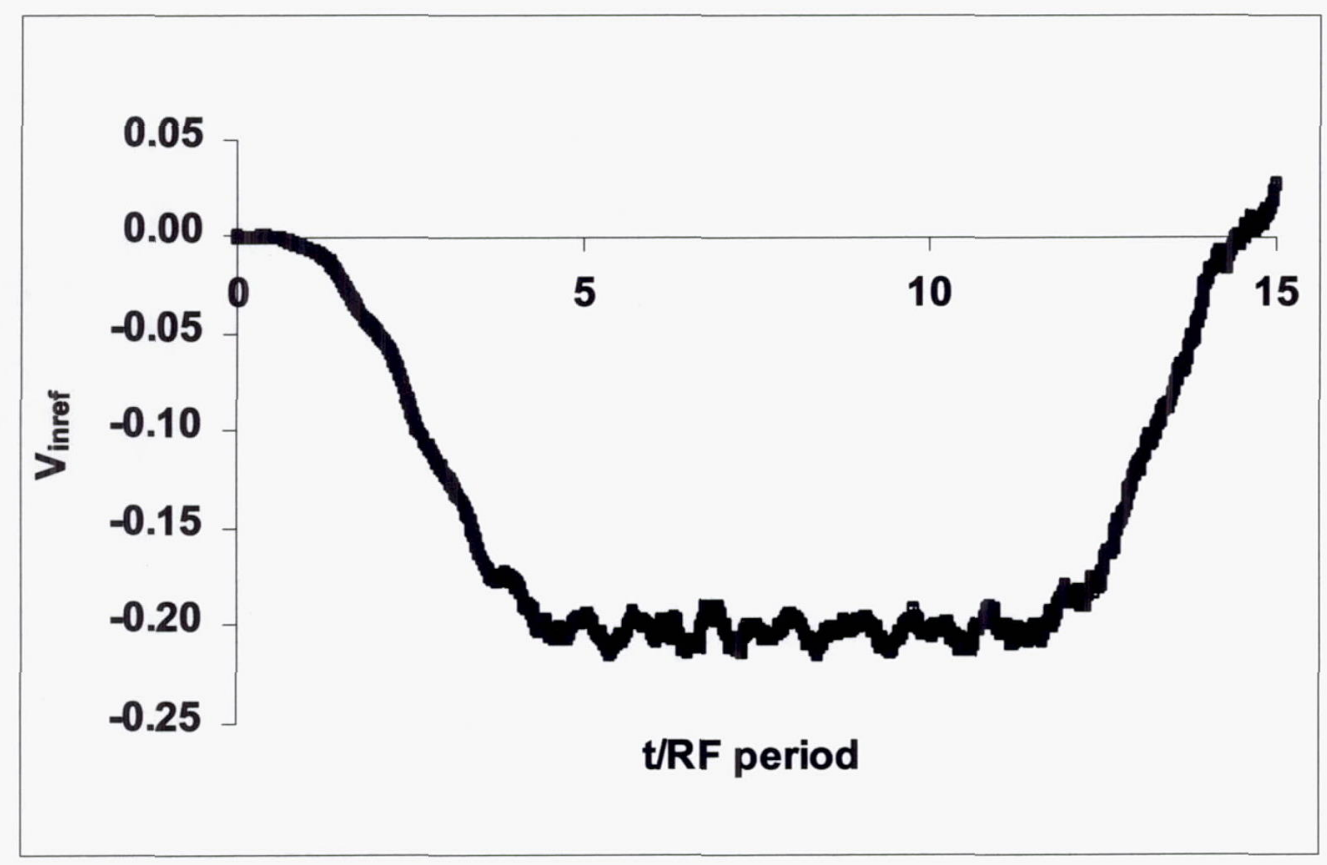

(b)

Figure 5 MAFIA time-domain signals for $P_{i n}=25 \mathrm{~mW}, f=29 \mathrm{GHz}$ and $\mathrm{b} / \mathrm{a}=\mathbf{0 . 5}$ (a) input and output signals (b) reflected signal at the input port 


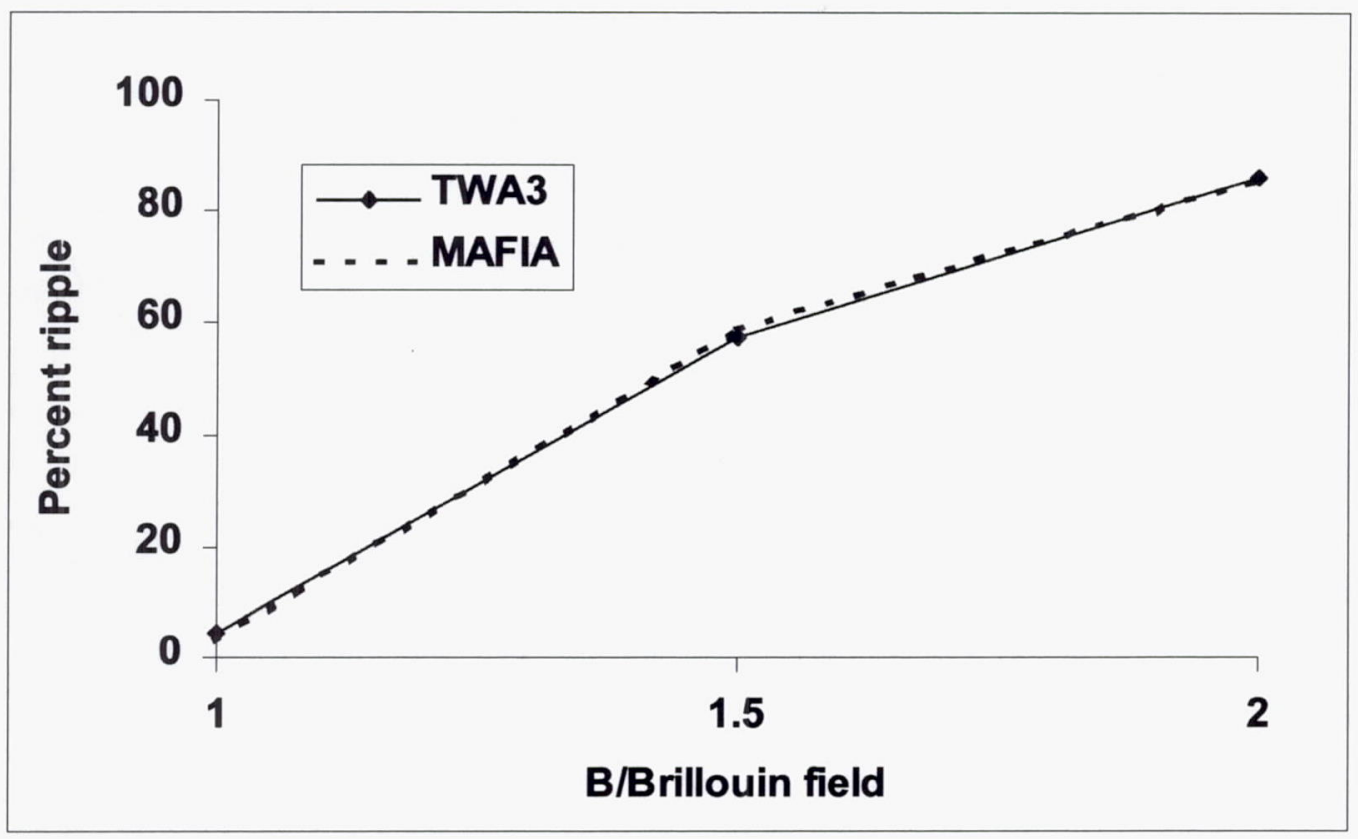

Figure 6 Simulated percent ripple using various focusing strengths for MAFIA and TWA3 models

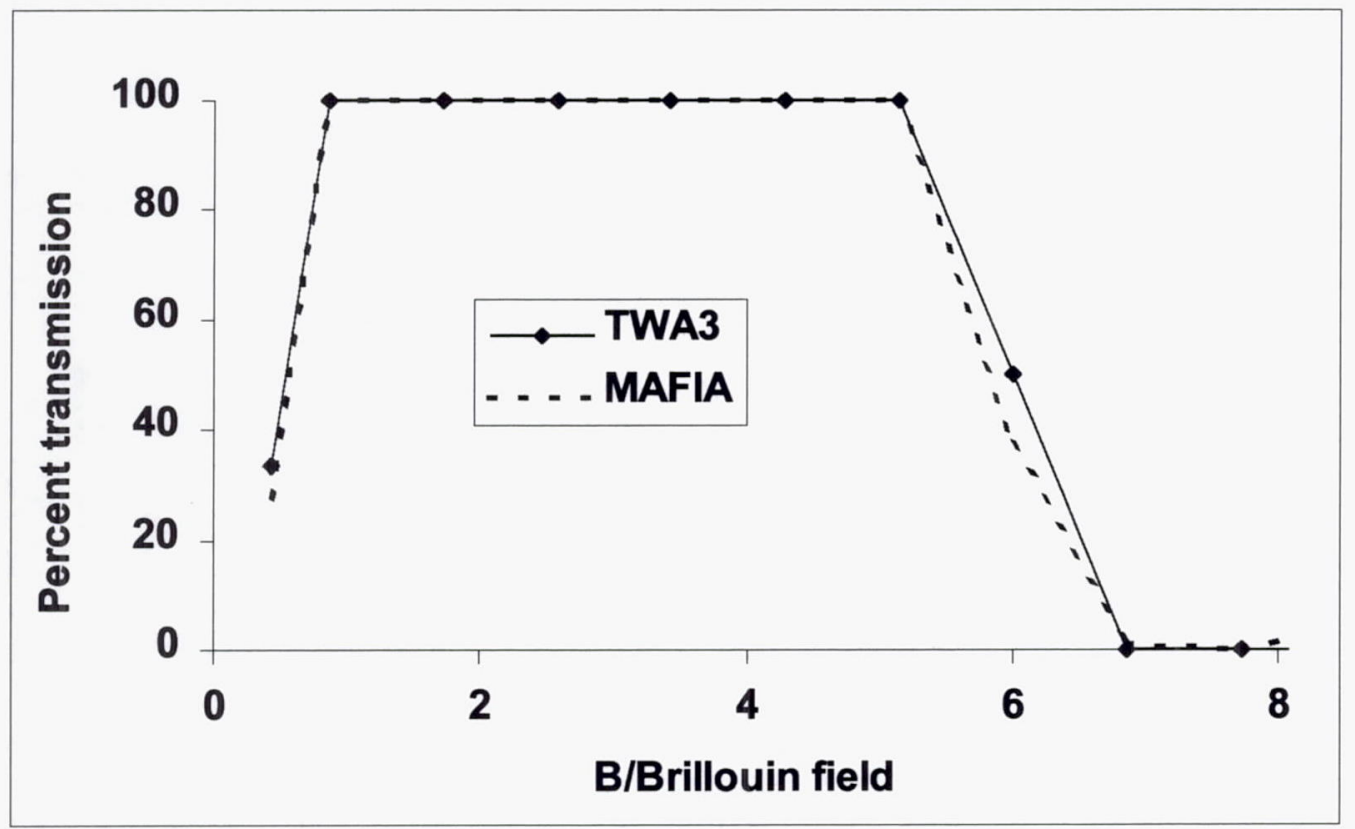

Figure 7 Simulated percent transmission using various focusing strengths for MAFIA and TWA3 models 


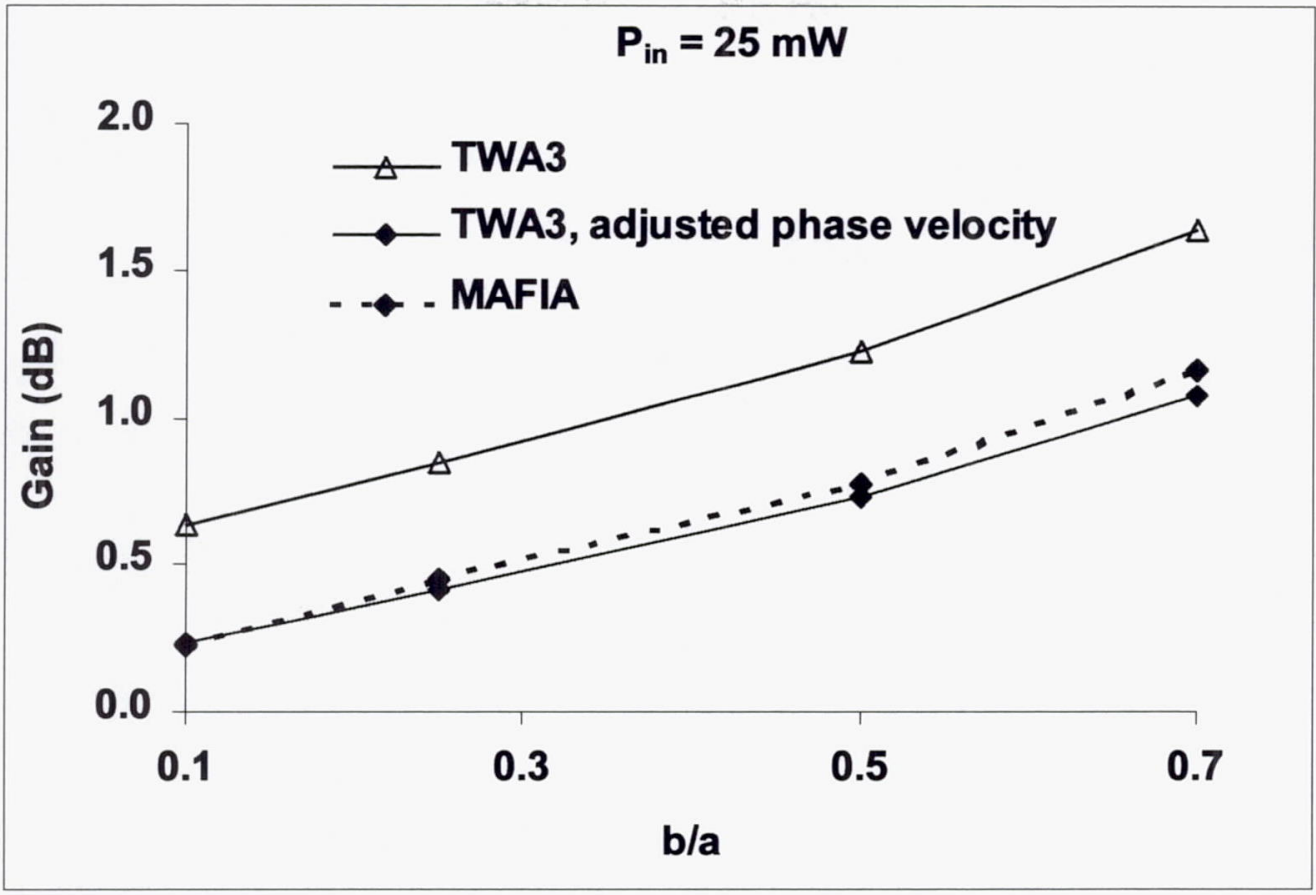

Figure 8 Gain as a function of beam radius for TWA3 and MAFIA models with $P_{\text {in }}$ $=25 \mathrm{~mW}$

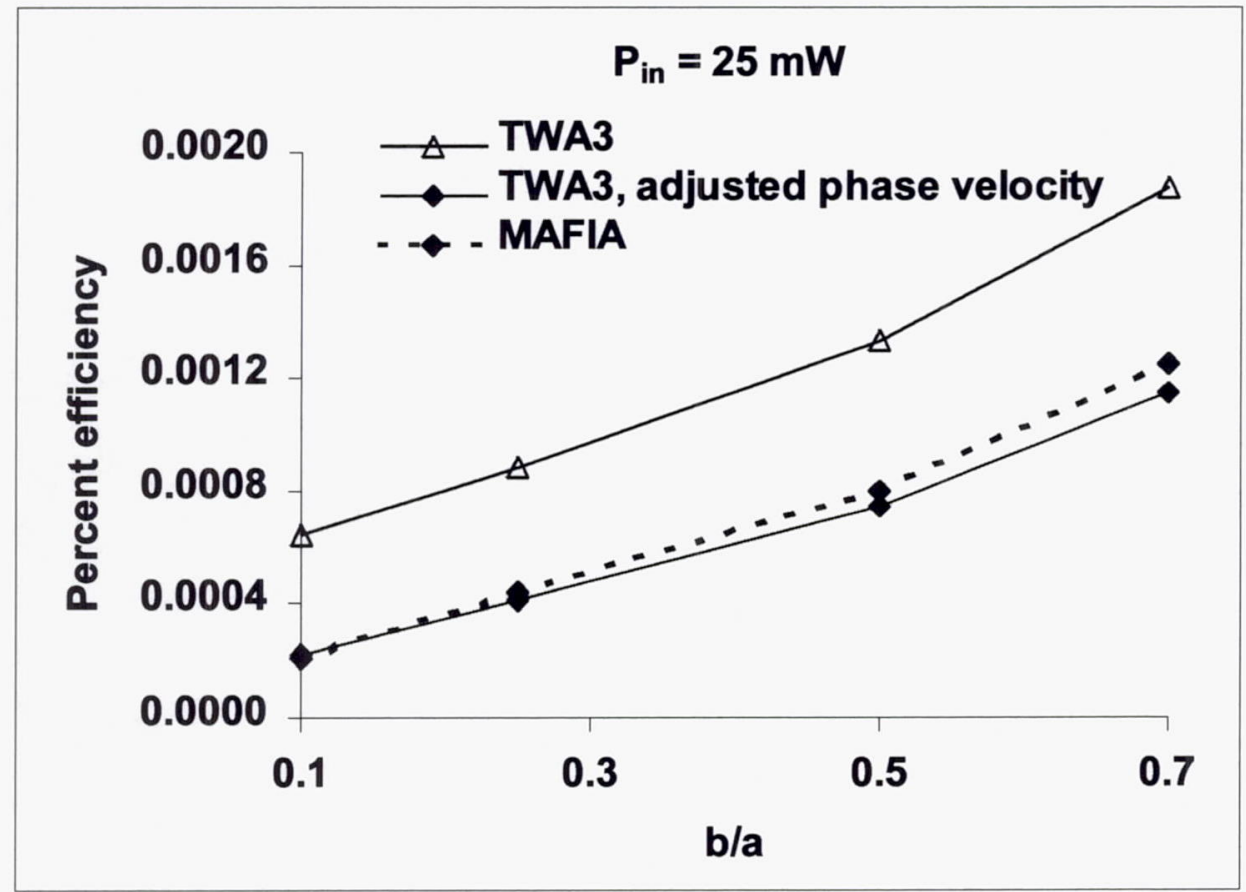

Figure 9 Efficiency as a function of beam radius for TWA3 and MAFIA models with

$$
P_{\text {in }}=\mathbf{2 5} \mathbf{m W}
$$



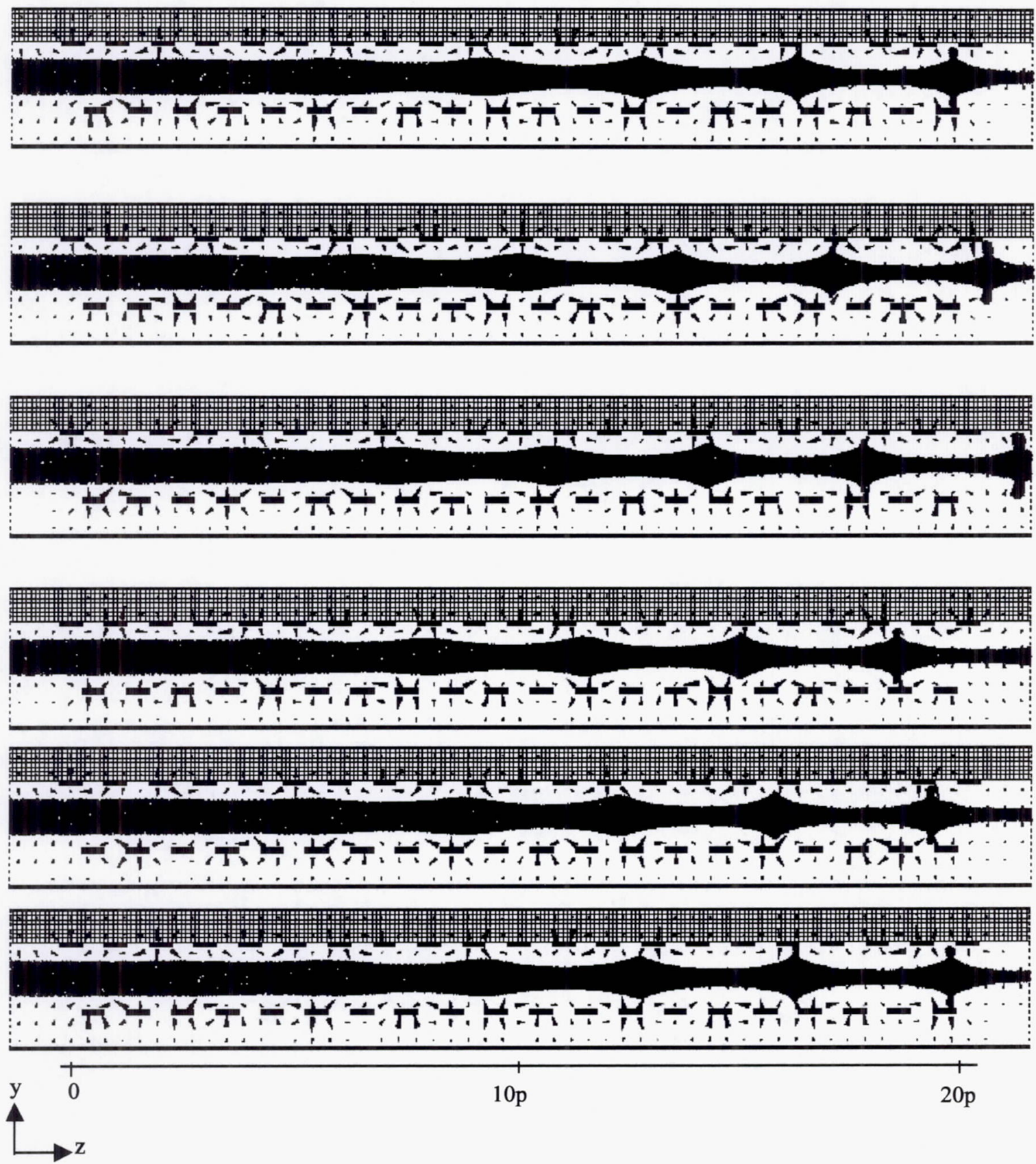

Figure 10 Simulated 2D MAFIA profiles of particles and dynamic electric fields at six time steps over one $R F$ period with $P_{\text {in }}=46 \mathrm{~W}$ and $b / a=0.5$ 


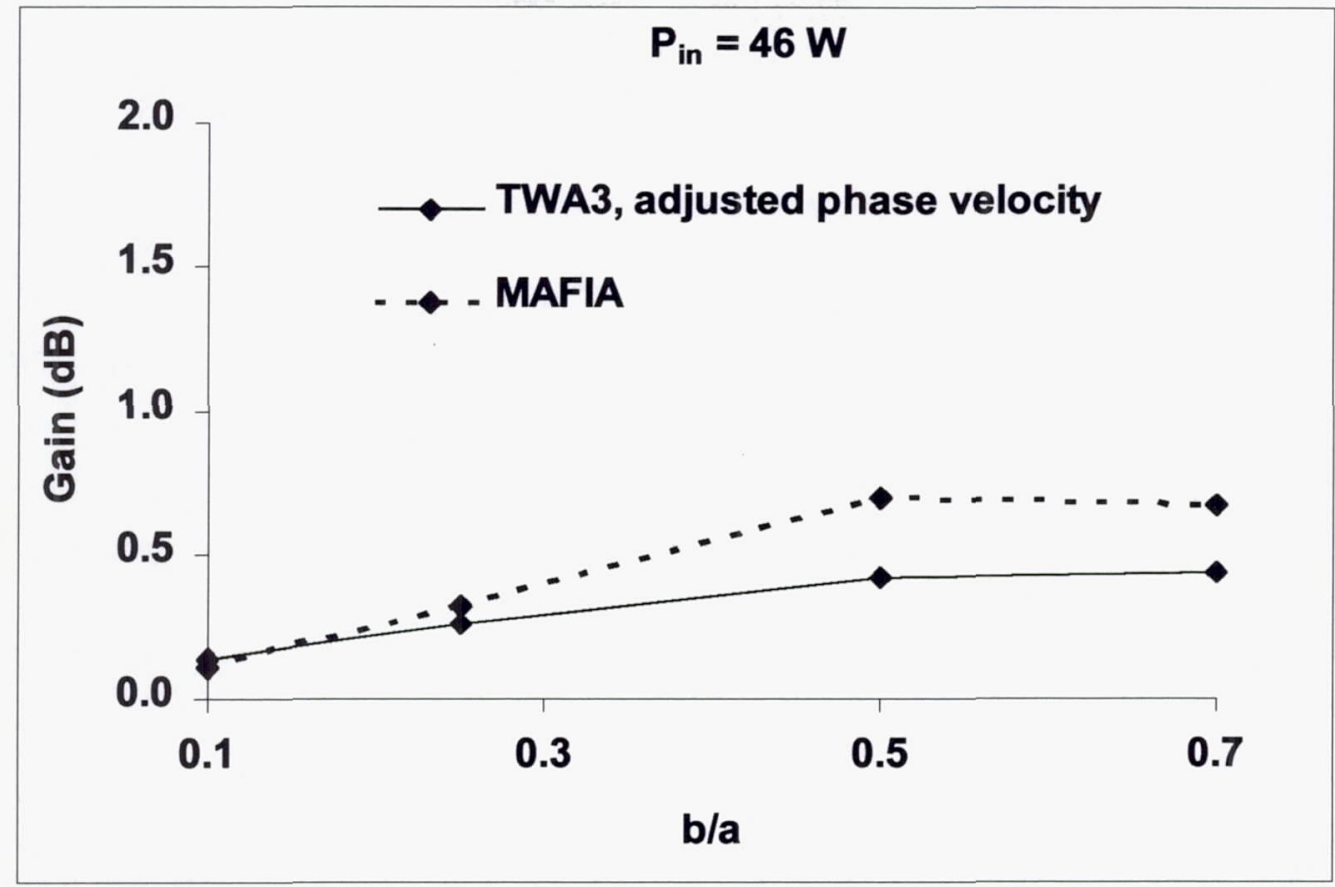

Figure 11 Gain as a function of beam radius for TWA3 and MAFIA models with $P_{\text {in }}$ $=46 \mathrm{~W}$

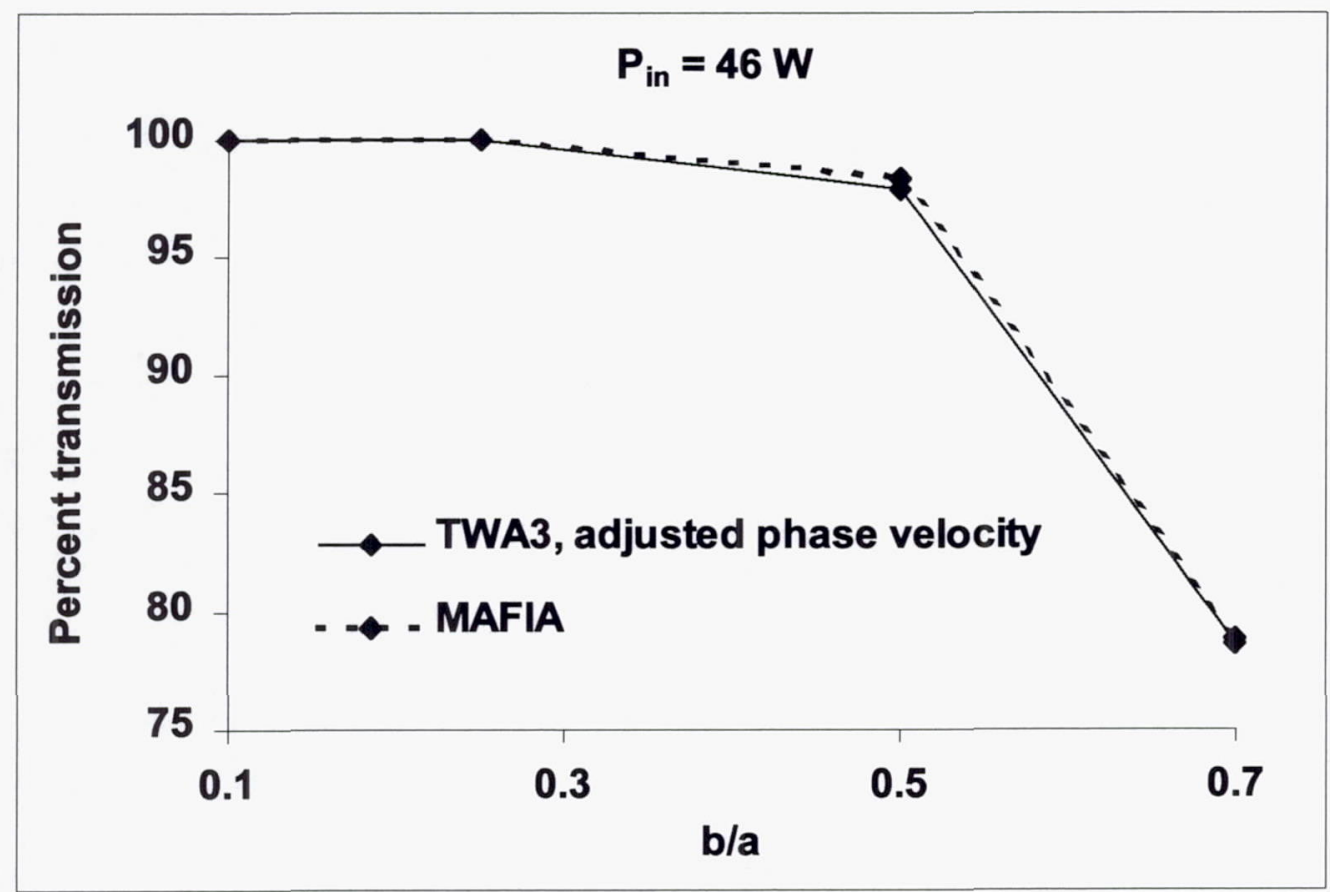

Figure 12 Percent transmission as a function of beam radius for TWA3 and MAFIA models with $P_{\text {in }}=46 \mathrm{~W}$ 


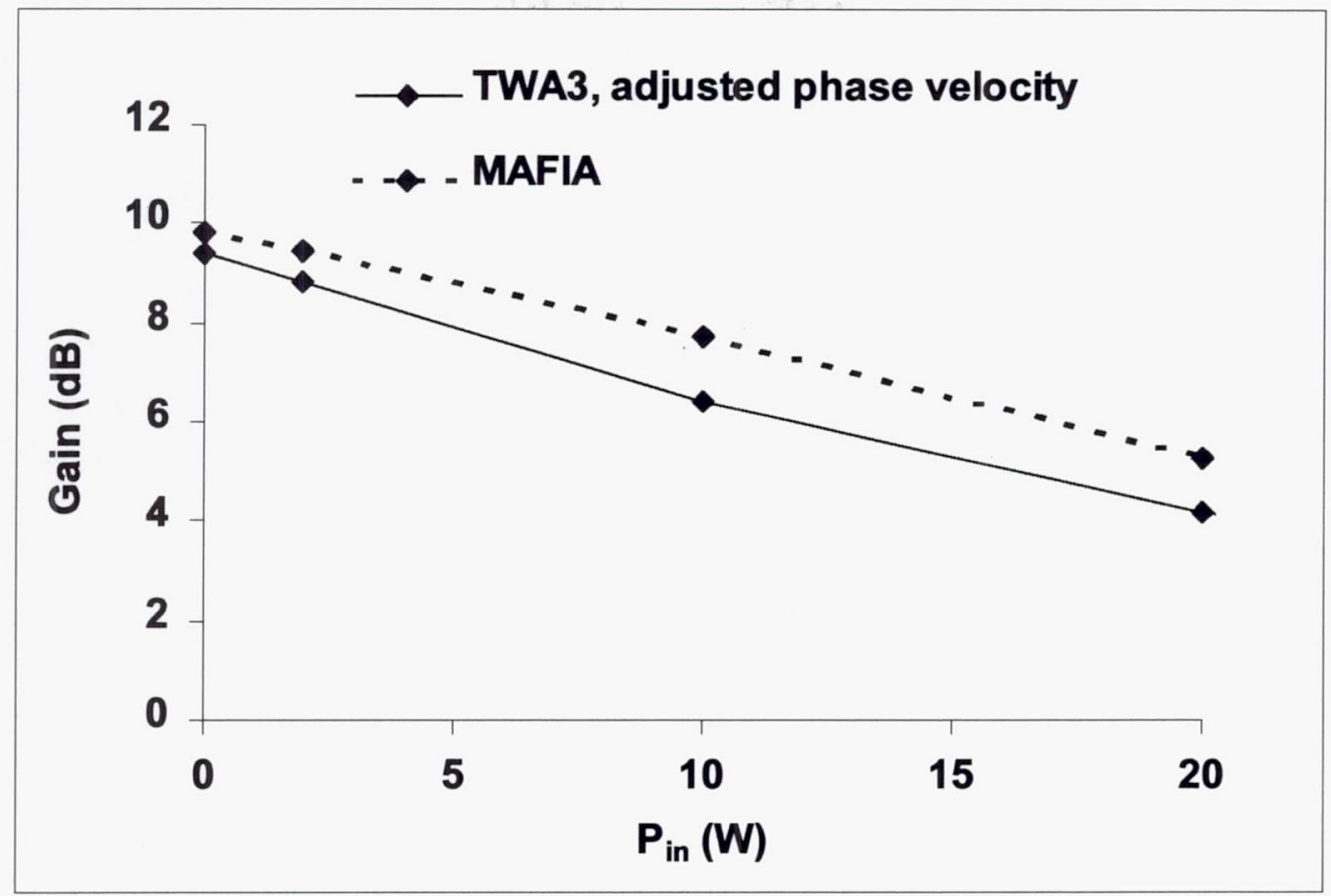

Figure 13 Gain as a function of input drive power for TWA3 and MAFIA 40 helical turn models with $b / a=0.5$

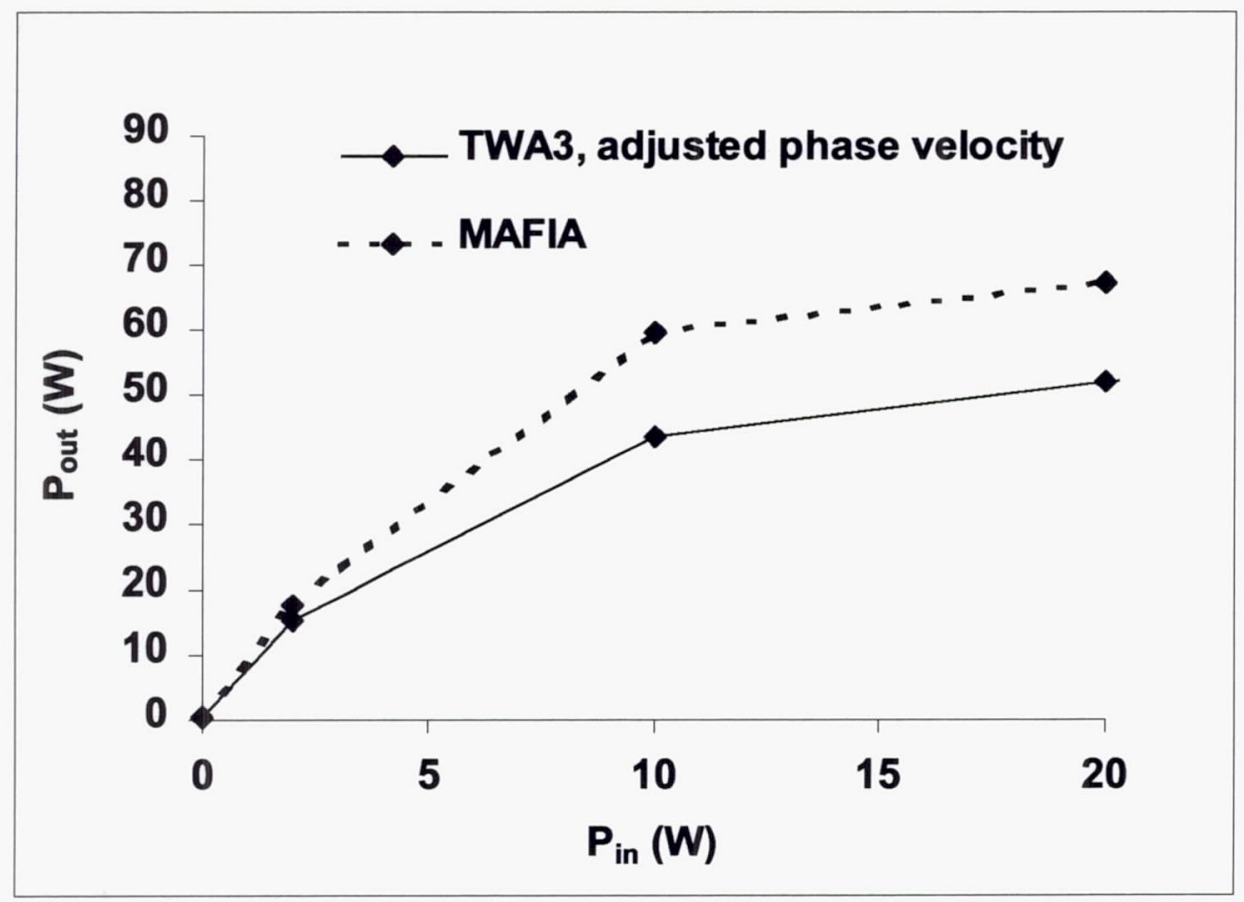

Figure 14 Output power as a function of input drive power for TWA3 and MAFIA 40 helical turn models with $b / a=0.5$ 


\section{REFERENCES}

i C. L. Kory and J. A. Dayton, Jr., Accurate cold-test model of helical TWT slow-wave circuits, IEEE Trans. on Electron Devices, Vol. 45, No. 4, pp. 966-971, April 1998. ii Personal communication with James A. Dayton, Jr. of Hughes Electron Dynamics, April 2000.

iii. T. Weiland, On the numerical solution of Maxwell's equations and applications in the field of accelerator physics, Part. Accel., Vol. 15, pp. 245-292, 1984.

iv. T. Weiland, On the unique numerical solution of Maxwellian eigenvalue problems in three dimensions, Part. Accel., Vol. 17, pp. 227-242, 1985.

v C. L. Kory and J. A. Dayton, Jr., Computational investigation of experimental interaction impedance obtained by perturbation for helical traveling-wave tube structures, IEEE Trans. on Electron Devices, Vol. 45, No. 9, pp. 2063-2071, September 1998.

vi C. L. Kory and J. A Dayton, Computational investigation of helical traveling wave tube transverse RF field forces, IEEE Trans. on Electron Devices, Vol. 47, No. 1, pp. 253-256, January 2000.

vii The MAFIA Collaboration, MAFIA TS3 the 3D-PIC Solver, December 91996.

viii D. M. MacGregor, Two-dimensional nonlinear multisignal helix traveling-wave tube amplifier computer program, Volume 1: User Manual, Electrocon International, Inc., Ann Arbor, Michigan, April 1993.

ix Personal communication with Will Menninger of Hughes Electron Dynamics, August 1997.

x Personal communication with Xiaoling Zhai of Hughes Electron Dynamics, June 1998. xi C. L. Kory, Three-dimensional simulations of PPM Focused Helical Traveling-wave tubes, Doctor of Engineering Dissertation, Cleveland State University, Cleveland, Ohio, August 2000.

xii J. T. Mendel, C.F. Quate and W. H. Yocom, Electron Beam Focusing with Periodic Permanent Magnet Fields, Proc. IRE, pp. 800-810, May 1954.

xiii C. L. Kory, Effect of Geometric Azimuthal Asymmetries of PPM Stack on Electron 
Beam Characteristics, Accepted for publication in IEEE Trans. on Electron Devices, January 2001.

xiv Personal communication with Jeff Wilson of NASA Glenn Research Center, February 2000.

xv K. Shalkhauser and G. Fujikawa, Bit-error-rate testing of high power $30 \mathrm{GHz}$

traveling wave tubes for ground terminal applications, NASA Technical Report 2635,

October 1986. 